\title{
Basement membrane
}

\author{
JOHN T. IRELAND \\ From the Southern General Hospital, Glasgow, and the University of Glasgow
}

Basement membrane has attracted less attention than the other components of connective tissue. But, like the other extracellular fibres and matrix materials, it can profoundly influence both the structure and function of advanced life forms. Usually taking the shape of thin, structureless cementing material between epithelial and connective tissue, basement membranes are widely distributed throughout the body. They are found as extracellular components of blood capillaries, renal glomeruli and tubules, alveoli, retina, lens capsule, muscle parasarcolemma, sweat glands, Schwann cells, and breast ducts. Although sharing similar morphological, biochemical, and antigenic properties, basement membrane can be developed for different functions in special situations. In the lens capsule of the eye, for example, it provides elastic support while in the glomerulus it is the foundation of selective filtration. Elastic support and filtration represent the essence of basement membrane function.

Because structure and function can be examined in detail in the glomerulus, it is an ideal model system in which to study the characteristics of basement membrane (Fig. 1). Moreover, the glomerulus is especially suited to detailed examination because cross-sectional measurements of basement membrane thickness are more precise in glomerular than in muscle or other capillaries (Cameron et al., 1975). Many of the recent biochemical studies have been of glomerular capillaries (Spiro, 1976). Further insight derives from examining the defects associated with glomerular capillary disease. In particular, the metabolic changes in the diabetic glomerulus show how alteration in the biochemical configuration of basement membrane not only modifies filtration but leads to the deposition of macromolecules such as fibrin. Mechanisms leading to fibrin or antigen deposition are obviously relevant to the development of connective tissue disease.

Accordingly, this review will focus on the glomerular model, examining normal basement membrane turnover and biochemical structure. The metabolic changes in diabetes that lead to altered basement membrane porosity and fibrin deposition will be reviewed with the aim of providing new insight into the function of basement membrane in health and disease.

\section{Basement membrane turnover}

Much evidence has accumulated from various sources (Farquhar et al., 1961; Andres et al., 1962; Kurtz and Feldman, 1962; Vernier, 1964; Pierce, 1966; Thoenes, 1967; Lee et al., 1969; Walker, 1973) to show that visceral epithelium is responsible for basement membrane synthesis (Fig. 2). Until recently, however, its rate of turnover had been less clear. The elegant, long-term sequential studies of Walker (1973), using the argyric technique, confirmed the view that turnover is slow. Clearance of silver from the rat glomerulus is exclusively undirectional from the outer epithelial aspect. No reverse movement is observed. In mature animals the turnover of base-

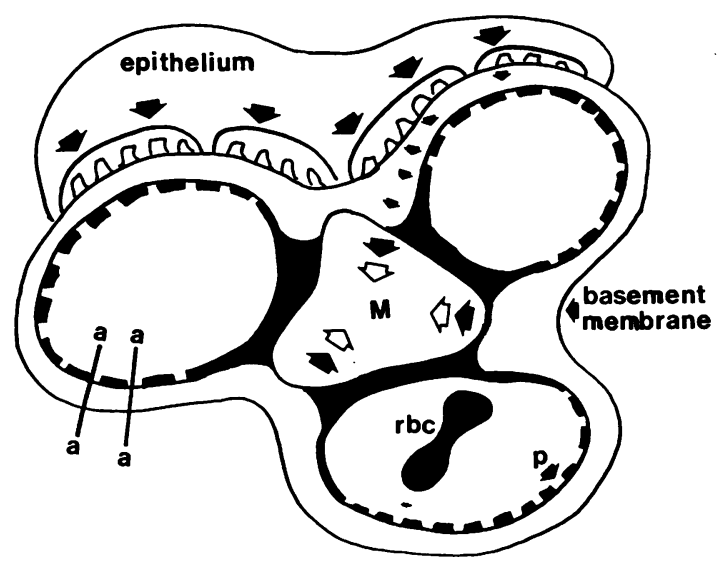

Fig. 1 Diagram of glomerular capillary lobule. Three capillaries cut in cross section with red blood cell $(\mathrm{rbc})$ within the capillary lumen of one to indicate scale. Epithelium attached to capillary basement membrane by foot processes. Mesangial cell $(M)$. Direction of basement membrane turnover from epithelium to mesangium indicated by arrows. Cross section thickness of membrane measured as at aa, aa. 


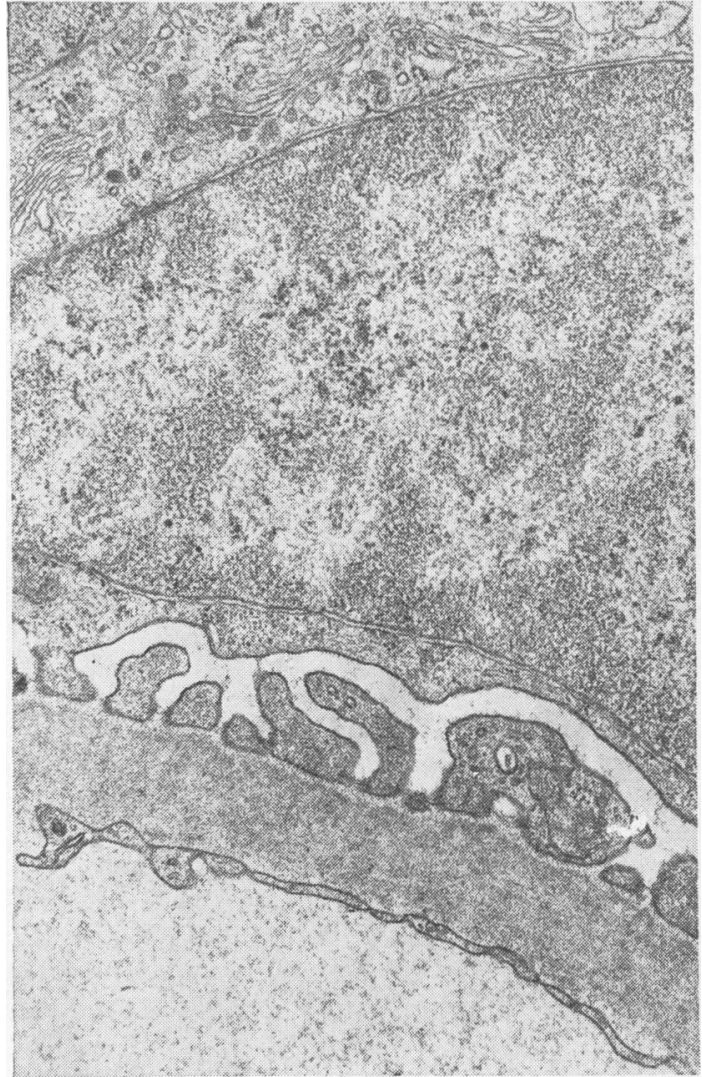

Fig. 2 Epithelial cell (above) attached to capillary basement membrane by system of foot processes. $(40 \times 000)$

ment membrane from the epithelial to the endothelial surface takes about one year. This investigation has also illuminated basement membrane removal. Walker (1973) showed not only a slow and continuous addition of new basement membrane on the epithelial aspect of the capillary but also a simultaneous removal from the endothelial side, where it was phagocytosed by mesangial cells (Fig. 3). This evidence confirms the earlier postulate of Farquhar (1964) that the mesangial cells have a phagocytic function. Although such studies suggest a slow, straightforward, and continuous process of basement membrane turnover from epithelium to mesangium, Walker (1973) has also postulated that there may be a second endothelial component in capillary basement membrane accounting for the electron-lucent lamina rara interna. This may not be a permanent component, however, since it disappears from the tissue of mature rats (Fox et al., 1977) and is not evident in the adult human glomerulus (Fig. 2).

How is basement membrane removed from nonglomerular tissue devoid of mesangial cells? Polymorphonuclear leucocytes contain neutral proteases capable of extensively digesting vascular basement membrane (Brownlee, 1976) and possibly playing a significant part in turnover in the glomerulus and elsewhere. Proteases may have particular significance in diseases where an increased concentration of $\alpha_{2}$ macroglobulin or of $\alpha_{1}$-antitrypsin may inhibit leucocyte neutral proteases, retarding basement membrane removal.

Diseases associated with excess basement membrane may arise from increased epithelial synthesis, from impaired removal, or from variable combination of both processes. The slow turnover of basement membrane has two important implications. Firstly, analysis of its composition in short-term investigations of experimental diseases such as alloxan- or streptozotocin-diabetes may give misleading results. Examination of tissue within 12 months inevitably means that residual normal tissue will be present. Secondly, slow turnover suggests that antibody to basement membrane, or other antigenantibody complexes, may remain within the basement membrane for a considerable time.

\section{Basement membrane biochemistry}

Histochemical staining with the periodic acid-Schiff reaction showed the light microscopist that basement membranes were a dehydrated gel of polysaccharide and protein, described as 'mucopolysaccharides'. This terminology was adequate so long as the precise constituents remained uncertain. Improved techniques of isolation by ultrasonic disruption and differential centrifugation have shown that the basement membrane material belongs to the collagen group of glycoproteins and contains the aminoacids characteristic of collagen (glycine, hydroxylysine, and hydroxyproline) in relatively large amounts. D. S. Jackson and A. J. Bailey comprehensively review collagen synthesis in their papers at pages 44 and 49 respectively (Jackson, 1978; Bailey, 1978).

Whether basement membrane contains collagen is still disputed. Although no typical collagen fibres of $640 \AA$ periodicity can be detected in normal basement membrane, Kefalides $(1973,1974)$ has been able to use enzymic dissection techniques to isolate a discrete type IV collagen. This evidence is disputed by Spiro (1976), who argues that this 'collagen' is not a natural component of basement membrane but probably represents a minor fragment resistant to degradation, consequent on defects in proteolytic digestive technique. By a combination of gel filtra- 


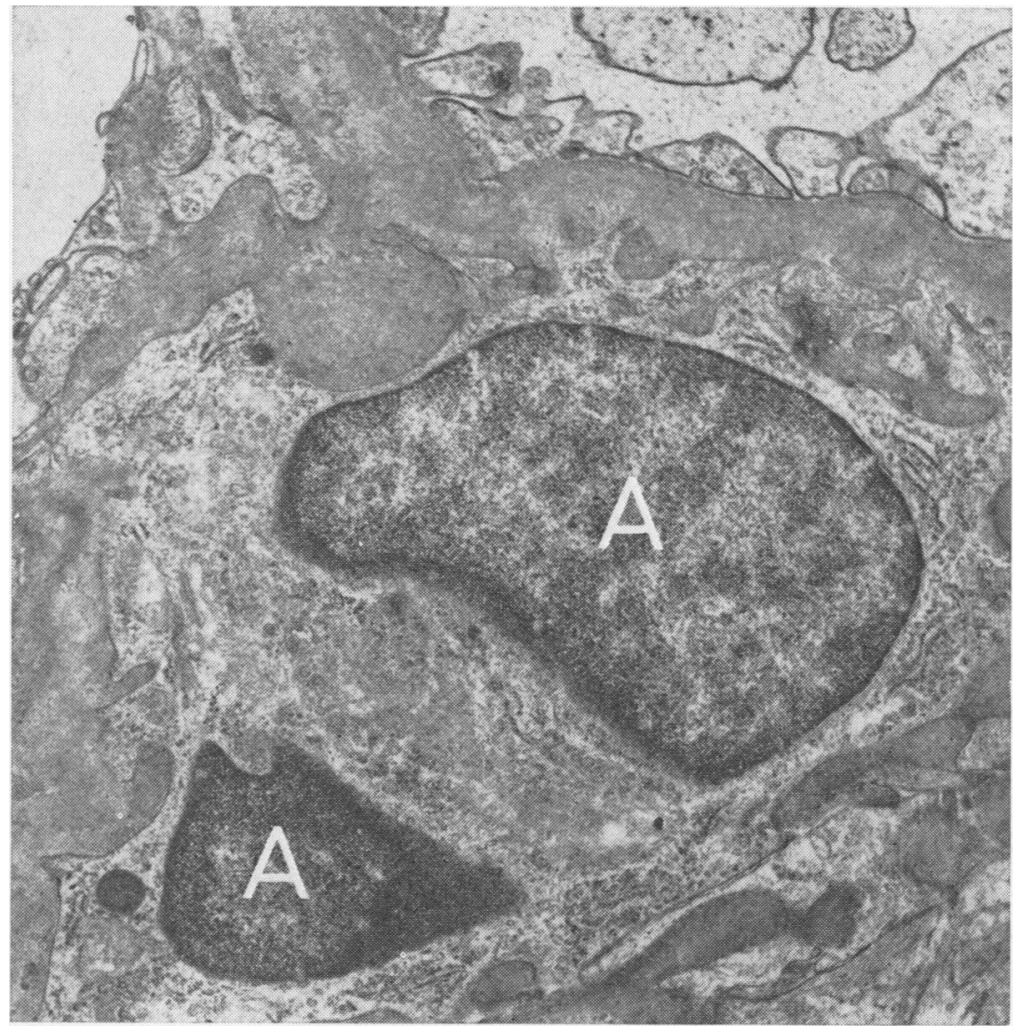

Fig. 3 Mesangial cell showing nuclear tissue $(A)$ and cytoplasm engulfing basement membrane material. $(\times 20000)$

tion, ion exchange chromatography, and polyacrylamide gel electrophoresis Spiro (1976) has developed an alternative view of basement membrane glycoprotein biochemistry. Not only did peptide subunits vary greatly in molecular weight ( 25000 to 220000 daltons) but they also differed in their content of the amino-acids characteristic of collagen. Most subunits contained carbohydrate in variable proportions as indicated by their hydroxylysine and glucosamine contents. Spiro (1976) concluded that subunit polydispersity is one of the most striking structural features of basement membrane. Subunit polydispersity does not imply that the various peptides represent different biosynthetic steps in the ribosomes of epithelial cytoplasm. Instead, the variations may well be a consequence of limited physiological proteolysis by the neutral proteases of polymorphonuclear leucocytes (see above under 'Basement membrane turnover') or by enzymes from glomerular mesangial cells.

Whatever the outcome of arguments about the presence or absence of collagen the question reremains: Can we learn more about basement membrane function (and dysfunction) by studying its glycoproteins in greater detail ? Further examination of the carbohydrate component may be relevant to our understanding of permeability and alterations in filtration.

Carbohydrate forms about $10 \%$ of basement membrane glycoprotein (Beisswenger and Spiro, 1970; Spiro, 1976). This relatively high content is another feature differentiating basement membrane from fibrillar collagen. The carbohydrate constituents are distributed almost evenly by weight between two distinct types of saccharide units. One unit is a disaccharide (glucose-galactose-hydroxylysine) with a $\beta$-glycosidic bond linking galactose to hydroxylysine. There are 10 such units of molecular weight 324 daltons to each of the other large units of 3200 daltons. The other carbohydrate unit is a branched hetero-polysaccharide consisting of sialic acid, fucose, galactose, mannose, and $\mathrm{N}$-acetylglucosamine arranged in a branching sequence with many incomplete residues present (Alberti and Hockaday, 1975; Spiro, 1976).

According to Spiro (1976) the importance of these numerous bulky carbohydrate components may lie in the way the peptide chains pack together. The 
amorphous nature of the membrane may be due to the steric hindrances which the closely spaced saccharide units exert on fibril formation. In other words, the saccharide units or carbohydrate components may hold the peptide chains apart and determine porosity. Spiro (1976) has visualised basement membrane as a cross-linked structure in which the peptide chains are layered over each other with disulphide bonds as the major interchain links (Fig. 4).

\section{Metabolism of glomerular basement membrane in diabetes and hormonal imbalance}

It is now beyond dispute that long-standing diabetes mellitus leads to thickening of the glomerular capillary basement membrane (Fig. 5) and to an increased tendency to proteinuria (Cameron et al., 1975). What can we learn about the structure and function of basement membrane by examining this metabolic disorder?

Analysis of human diabetic glomerular capillary basement membrane shows an absolute increase in glycoprotein. Kefalides (1973) found little significant alteration in composition, while Spiro (1976) found an increase in the glucose and galactose components linked to increased hydroxylysine. The increase in hydroxylysine is matched by a reciprocal fall in the lysine content. An interesting question arises-if the carbohydrate components maintain normal porosity might these bulky carbohydrate components of diabetes lead to increased porosity and proteinuria?

There is now experimental evidence, derived from rats treated for long periods with streptozotocin, of a close correlation between control of blood glucose and basement membrane thickening (Fox et al., 1977). How may poor diabetic control (that is, chronic hyperglycaemia) affect basement membrane synthesis? Increased glucose concentration in diabetes is freely permeable in kidney tissue independent of insulin. Moreover, the enzyme responsible for the incorporation of carbohydrate into the hydroxylysine-linked disaccharide, glucosyltransferase, is increased in experimental diabetes (Spiro and Spiro, 1971). From this evidence and from the known effect of growth hormone on peptide synthesis in epithelial cells Alberti and Hockaday (1975) postulate that the thickened basement membrane of diabetes depends on the high levels of growth hormone (Lundbaek et al., 1970) and of glucose in this disorder.

There are other diseases in which the hormones of stress (cortisol, growth hormone, glucagon, and catecholamines) are secreted in excess. Is it possible they may have a similar influence on basement membrane metabolism, favouring incorporation of

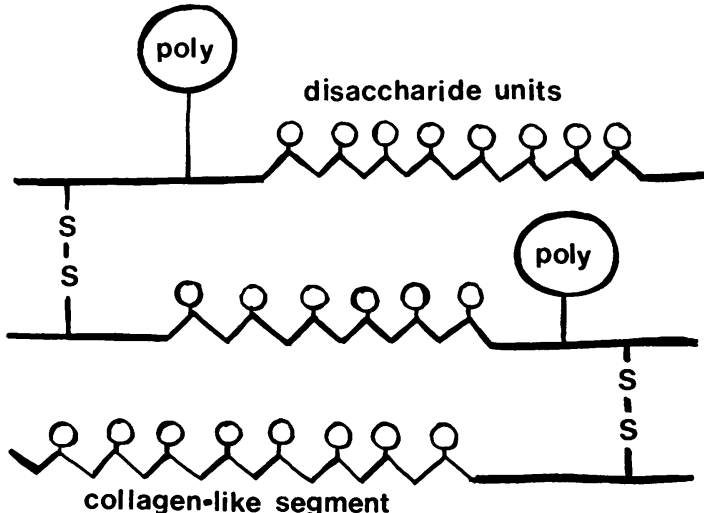

Fig. 4 Diagram of peptide chains of glomerular basement membrane joined by disulphide $(S-S)$ bonds. Irregular lines represent helical (collagen-like) segments with small disaccharide units attached. Larger polysaccharide units (POLY) attached to straight polar segments.

glucose into bulky carbohydrate components and increasing membrane porosity?

\section{Altered basement membrane function: fibrin and antibody deposition}

The biochemical and other evidence (Larsson, 1967; Balodimos et al., 1971) suggests that the altered structure of basement membrane may affect its permeability. In view of the increasing importance of fibrinogen and related products in the pathogenesis of various human and experimental glomerulopathies (McCluskey and Vasalli, 1969; Clarkson et al., 1971), the finding by immunofluorescence and electron microscopy (Farquhar et al., 1972) of fibrin deposition in the basement membrane of diabetics may represent a significant consequence of altered membrane porosity (Figs. 6, 7). The concept of altered vascular permeability, with early mural infiltration by mesometric substances, including fibrinogen, has received little support until recently, yet Lendrum $(1963,1969)$ has consistently implicated fibrin in 'plasmatic vasculosis'.

The altered permeability of basement membrane in such metabolic disorders as diabetes may mislead the immunologist. Thus Westberg and Michael (1972), in a detailed study of the immunohistopathology of diabetic glomerulosclerosis, demonstrated a thin linear staining of basement membrane for IgG and $\operatorname{IgM}$ in about $50 \%$ of cases. Reactions for fibrin, $\mathrm{B}_{1} \mathrm{C}$, albumin, and ceruloplasmin were less often positive. However, heterologous $\mathrm{C}^{1}$ did not bind in vitro to the basement membrane. 


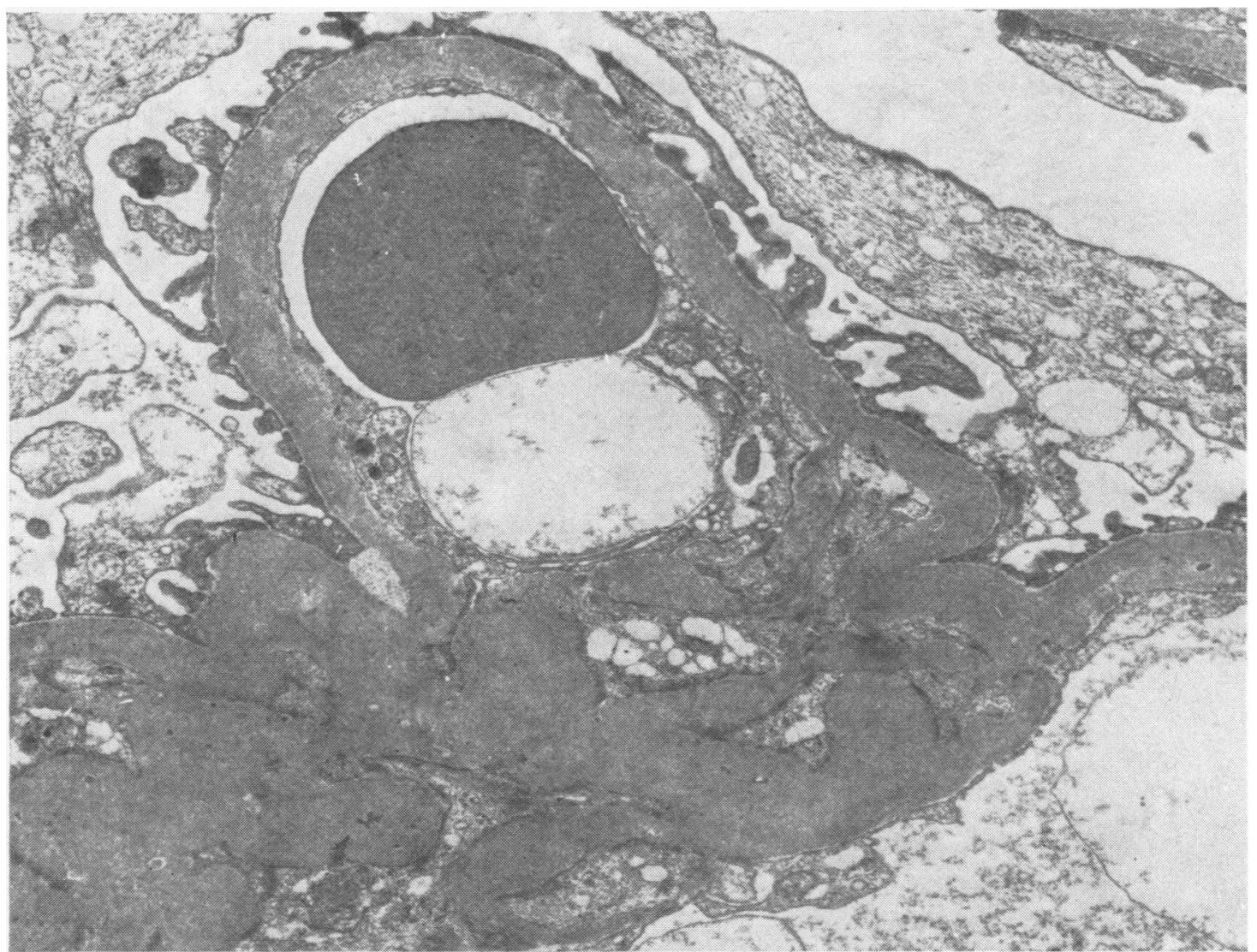

Fig. 5 Capillary loop in long-standing diabetic showing red blood cell within lumen and general thickening of basement membrane. Accumulation of basement membrane material within mesangial stalk (lower part of plate). $(\times 12000)$

Fluorescent insulin did not bind to diabetic membrane and antibodies to insulin did so only exceptionally. These workers concluded that serum proteins were found in the basement membrane possibly as a consequence of altered permeability rather than as a result of immunological mechanisms.

Evidently the immunologist must always be cautious, particularly in disorders associated with proteinuria and increased basement membrane permeability, that immunofluorescent and other techniques are not simply demonstrating abnormal proteins that have been trapped in a leaking membrane. Antibodies to either endogenous or exogenous non-basement-membrane antigens take part in forming circulating immune-complexes which can be passively trapped in the basement membrane in various forms of glomerulonephritis (Wilson and Dixon, 1972). Such antibodies are randomly and irregularly deposited in the basement membrane. In contrast, antibodies to specific determinants in basement membrane are distributed in a smooth, continuous, linear fashion along the entire membrane.

\section{Conclusion}

The origin, turnover, biochemical configuration, and permeability of basement membrane have been examined with particular reference to the glomerulus. Arising from the visceral epithelial cell, basement membrane turns over extremely slowly. Ultimately it is removed by the neutral proteases of leucocytes or of mesangial cells. Chemically it belongs to the collagen group of glycoproteins, but no collagen fibres are visible in its amorphous structure. There is a high content of carbohydrate $(10 \%)$ attached to the peptide chains either as small disaccharides or as larger polysaccharides. These carbohydrate units may be responsible both for the amorphous nature 


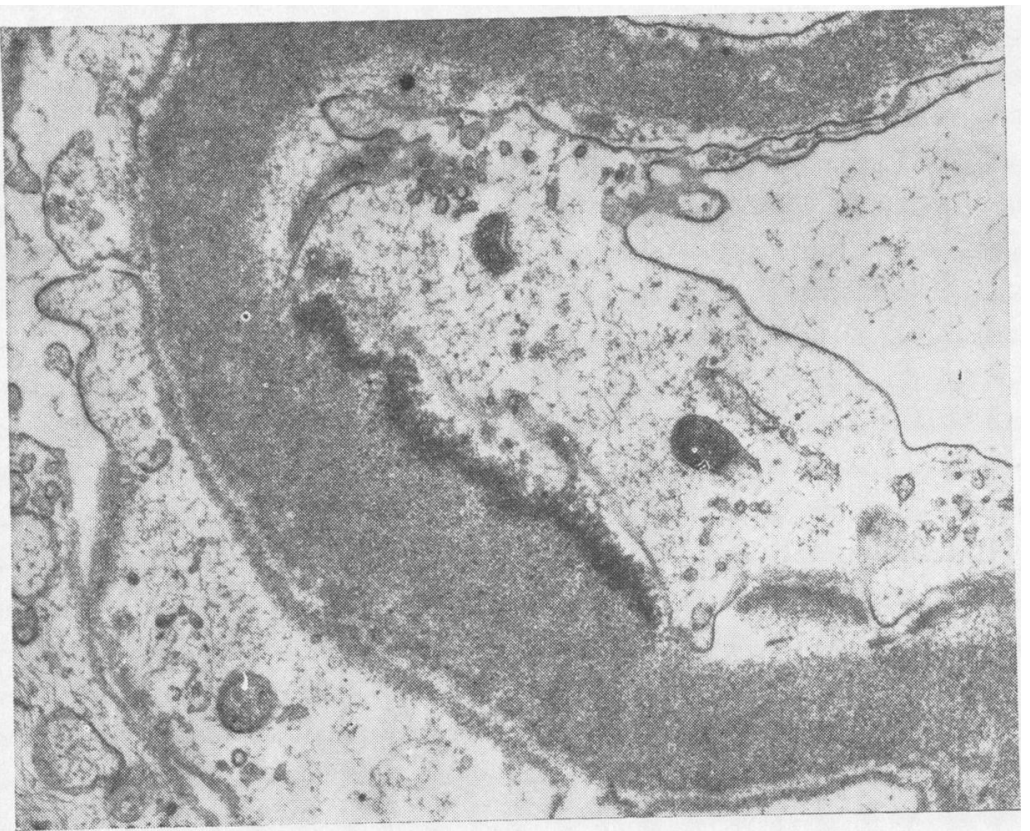

Fig. 6 Electron-dense granular material lying along the endothelial aspect of basement membrane in a diabetic patient. $(\times 28000)$

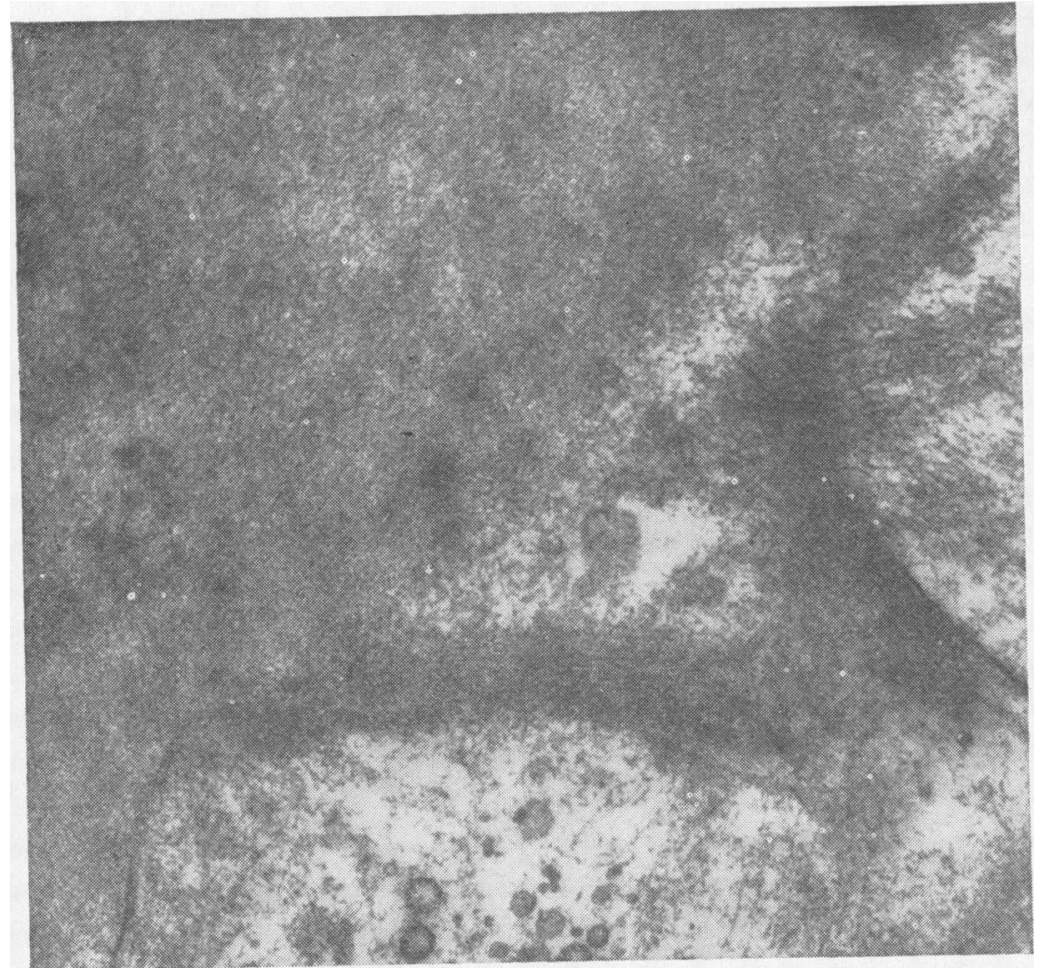

Fig. 7 Electron-dense fibrillar material on endothelial aspect of basement membrane in a diabetic patient. $(\times 52000)$ 
and for the permeability of the membrane by steric hindrance of fibril formation.

In the metabolic disturbance of diabetes not only is there gradual increase in the quantity of basement membrane, which is related to the duration of the disease and to hyperglycaemia, but bulky carbohydrate components on the peptide chain may increase permeability. Fibrinogen-derived macromolecules are then precipitated, leading to fibrin deposition and the more advanced changes of diabetic glomerulosclerosis. The hormones of stress (cortisol, growth hormone, and glucagon) may similarly modify the metabolism of the basement membrane in other chronic disease states. Fibrin, antigen-antibody complexes, or other macromolecular deposits within the basement membrane may have a bearing on changes produced in various connective tissue diseases. The slow turnover of basement membrane and the presence in chronic disease of factors such as increased circulating $\alpha_{2}-$ macroglobulins-which inhibit the neutral proteases that are capable of digesting the basement membrane-imply that repair processes will be slow.

Amorphous, unimpressive, and unexciting, nevertheless basement membranes provide research workers and histopathologists with a valuable mirror that reflects the nature of various disorders. Locked within the complex lattice structure of basement membrane are the secrets of many processes fundamental to our understanding of connective tissue and other diseases.

\section{References}

Alberti, K. G. M. M., and Hockaday, T. D. R. (1975). The biochemistry of the complications of diabetes mellitus. In Complications of Diabetes, edited by $\mathrm{H}$. Keen and R. J. Jarrett, pp. 221-264. Arnold, London.

Andres, G. A., Morgan, C., Hsu, K. C., Rifkind, R. A., and Seegal, Beatrice C. (1962). Electron microscopic studies of experimental nephritis with ferritin-conjugated antibody. Journal of Experimental Medicine, 115, 929-936.

Bailey, A. J. (1978). Collagen and elastin fibres. Journal of Clinical Pathology, 31, Supplement (Royal College of Pathologists) 12, 49-58.

Balodimos, M. C., Gleason, R. E., Kahn, C. B., Chlouverakis, C., Jarrett, R. J., Keen, H., and Soeldner, J. S. (1971). Urinary albumin excretion in the offspring of conjugal diabetics. Lancet, 2, 239-242.

Beisswenger, P. J., and Spiro, R. G. (1970). Human glomerular basement membrane. Chemical alterations in diabetes mellitus. Science, 168, 596-598.

Brownlee, M. (1976). $\alpha_{2}$-Macroglobulin and reduced basement membrane degradation in diabetes. Lancet, $1,779-780$.

Cameron, J. S., Ireland, J. T., and Watkins, P. J. (1975).
The kidney and renal tract. In Complications of Diabetes, edited by H. Keen and R. J. Jarrett, pp. 99150. Arnold, London.

Clarkson, A. R., MacDonald, M. K., Petric, J. J. B., Cash, J. D., and Robson, J. S. (1971). Serum and urinary fibrin-fibrinogen degradation products in glomerulonephritis. British Medical Journal, 3, 447451.

Farquhar, M. G. (1964). Glomerular permeability investigated by electron microscopy. In Small Blood Vessel Involvement in Diabetes Mellitus, edited by M. D. Siperstein, A. R. Colwell, and K. Meger, pp. 3438. American Institute of Biological Sciences, Washington.

Farquhar, A., MacDonald, M. K., and Ireland, J. T. (1972). The role of fibrin deposition in diabetic glomerulosclerosis: a light, electron and fluorescence microscopy study. Journal of Clinical Pathology, 25, 657-667.

Farquhar, M. G., Wissig, S. L., and Palade, G. E. (1961). Glomerular permeability. I. Ferritin transfer across the normal glomerular capillary wall. Journal of Experimental Medicine, 113, 47-66.

Fox, C. J., Darby, S. C., Ireland, J. T., and Sönksen, P. H. (1977). Blood glucose control and glomerular capillary basement membrane thickening in experimental diabetes. British Medical Journal, 2, 605-607.

Jackson, D. S. (1978). Collagens. Journal of Clinical Pathology, 31, Supplement (Royal College of Pathologists) 12, 44-48.

Kefalides, N. A. (1973). Biochemical properties of human glomerular basement membrane in normal and diabetic kidneys. In Vascular and Neurological Changes in Early Diabetes (Advances in Metabolic Disorders, Suppl. 2), edited by R. A. Camerini-Davalos and H. S. Cole, pp. 167-177. Academic Press, New York.

Kefalides, N. A. (1974). Biochemical properties of human glomerular basement membrane in normal and diabetic kidneys. Journal of Clinical Investigation, 53, 403-407.

Kurtz, S. M., and Feldman, J. D. (1962). Experimental studies on the formation of the glomerular basement membrane. Journal of Ultrastructural Research, 6, 1927.

Larsson, O. (1967). Studies of small vessels in patients with diabetes. A clinical, histological and immunohistochemical study of diabetic and nondiabetic subjects with special reference to the occurrence of various plasma proteins in the dermal vessel walls. Acta Medica Scandinavica Supplement, 480.

Lee, P. A., Blasey, K., Goldstein, I. J., and Pierce, G. B. (1969). Basement membrane: carbohydrates and x-ray diffraction. Experimental and Molecular Pathology, $10,323$.

Lendrum, A. C. (1963). The hypertensive diabetic kidney as a model of the so-called collagen diseases. Canadian Medical Association Journal, 88, 442-452.

Lendrum A. C. (1969). The validation of fibrin, and its significance in the story of hyalin. In Trends in Clinical Pathology: Essays in Honour of Gordon Signy, pp. 159187. British Medical Association, London.

Lundbaek, K., Jensen, V. A., Olsen, T. S., Ørskov, H., 
Christensen, N. J., Johansen, K., Hansen, A. P., and Østerby, R. (1970). Growth hormone and diabetic angiopathy. (Letter.) Lancet, 2, 472.

McCluskey, R. T., and Vasalli, P. (1969). Experimental glomerular diseasé. In The Kidney, edited by $\mathrm{C}$. Rouiller and A. F. Muller, Vol. 2, pp. 83-198. Academic Press, New York.

Pierce, G. B. (1966). The development of basement membranes of the mouse embryo. Developmental Biology, 13, 231-249.

Spiro, R. G. (1976). Search for a biochemical basis of diabetic microangiopathy. Diabetologia, 12, 1-14.

Spiro, R. G., and Spiro, M. J. (1971). Effect of diabetes on the biosynthesis of the renal glomerular basement membrane. Studies on the glucosyltransferase. Diabetes, 20, 641-648.

Thoenes, W. (1967). Endoplasmatisches Retikulum und 'Sekretkörper' in Glomerulum-Epithel der Säuger- niere. Zeitschrift für Zellforschung und mikroskopische Anatomie, 78, 561-582.

Vernier, R. L. (1964). Electron microscopic studies of the normal basement membrane. In Small Blood Vessel Disease in Diabetes Mellitus, edited by M.D. Siperstein, A. R. Colwell, and K. Meyer, p. 57. American Institute of Biological Sciences, Washington.

Walker, F. (1973). The origin, turnover and removal of glomerular basement membrane. Journal of Pathology, 110, 233-244.

Westberg, N. G., and Michael, A. F. (1972). Immunohistopathology of diabetic glomerulosclerosis. Diabetes, 21, 163-174.

Wilson, C. B., and Dixon, F. J. (1972). Immunological aspects of glomerulonephritis. In Renal Disease, edited by Sir Douglas Black, 3rd edition pp. 275-294. Blackwell, Oxford. 